\title{
Calculation of guided wave interaction with nonlinearities and generation of harmonics in composite structures through a wave finite element method
}

\author{
D. Chronopoulos ${ }^{\mathrm{a}}$ \\ ${ }^{a}$ Institute for Aerospace Technology \& The Composites Group, The University of Nottingham, NG7 2RD, UK
}

\begin{abstract}
The extensive usage of composite materials in modern industrial applications implies a great range of possible structural failure modes for which the structure has to be frequently and thoroughly inspected. Nonlinear guided wave inspection techniques have been continuously gaining attention during the last decade. This is primarily due to their sensitivity to very small sizes of localised damage. A number of complex transformation phenomena take place when an elastic wave impinges on a nonlinear segment, including the generation of higher and sub-harmonics. Moreover, the transmission and reflection coefficients of each wave type become amplitude dependent. In this work, a generic Finite Element (FE) based computational scheme is presented for quantifying guided wave interaction effects with Localised Structural Nonlinearities (LSN) within complex composite structures. Amplitude dependent guided wave reflection, transmission and conversion is computed through a Wave and Finite Element (WFE) method. The scheme couples wave propagation properties within linear structural waveguides to a LSN and is able to compute the generation of higher and sub-harmonics through a harmonic balance projection. A Newton-like iteration scheme is employed for solving the system of nonlinear differential equations. Numerical case studies are presented for waveguides coupled through a joint exhibiting nonlinear mechanical behaviour.

Keywords: Wave Interaction with Damage, Finite Elements, Composite Structures, Nonlinear Ultrasound, Structural nonlinearities
\end{abstract}

\section{Introduction}

Modern industrial structures are increasingly made of composite layered materials due to their well-known benefits. Composite structures may however exhibit a great variety of structural failure modes (including delamination, fibre breakage, matrix cracking and debonding) and must be frequently inspected in order to ensure continuous structural integrity. An increasing tendency within the Structural Health Monitoring (SHM) community is the 'shift to the left' maintenance strategy [1] for which the earliest possible detection of damage is important. When it comes to the aeronautical industry, approximately $27 \%$ of an average modern aircraft's lifecycle cost [2] is spent on inspection and repair. The use of 'offline' structural inspection techniques currently leads to a massive reduction of the

Email address: Dimitrios.Chronopoulos@nottingham.ac.uk (D. Chronopoulos) 


\section{Nomenclature}

$\mathcal{A}_{q}^{\omega,-}, \mathcal{A}_{f}^{\omega,-} \quad$ Generalised displacement and force wave interaction coefficients for an incoming wave of frequency $\omega$

$\mathbb{M}, \mathbb{C}$ and $\mathbb{K} \quad$ Mass, damping and stiffness matrices of the coupling element

D

Dynamics Stiffness Matrix (DMS) of a waveguide's modelled periodic segment

$R \quad$ Real operator

$\mathbf{R}$

Transformation matrix

S

Wave scattering coefficient matrix

$\mathbf{T}$

Wave propagation transfer matrix

$\mathbf{T}_{1}, \mathbf{T}_{2}$

Harmonic motion transformation matrices, functions of $\tau$

$\mathbf{a}^{+}, \mathbf{a}^{-}$

Amplitudes of waves moving towards and away of the coupling element

f

$\mathbf{f}_{N L}$

Forcing vector for an elastic waveguide

$\mathbf{q}$

Nonlinear force vector induced by the coupling element's inherent inelastic behaviour

Physical displacement vector for an elastic waveguide

$\mathbf{Z}$

Physical displacement vector for the coupling element

$L_{x}$

Dimension of a waveguide's modelled periodic segment

$L, R, I \quad$ Left, right sides and interior indices

c Transmission coefficient

$r \quad$ Reflection coefficient

$k \quad$ Wavenumber

$j$

$h, H$

Number of DoF on each cross-section of the periodic waveguide segment

$H_{m}$

Harmonic index and total number of harmonics considered in the harmonic balance projection

$n, N$

Index of the lowest subharmonic considered in the harmonic balance projection

$w, W_{n}$

Waveguide index and total number of waveguides existing in the considered system

Wave eigenvector index and total number of waves accounted for in waveguide $n$

$S$

Periodic segment positioning index

$t$

Time

$\boldsymbol{\Phi}_{q}^{h \omega,+}, \boldsymbol{\Phi}_{q}^{h \omega,-}$

$\boldsymbol{\Phi}_{f}^{h \omega,+}, \boldsymbol{\Phi}_{f}^{h \omega,-}$

Grouped displacement eigenvectors for the positive and negative going elastic waves at frequer cy $h \omega$

$\boldsymbol{\phi}_{q}, \boldsymbol{\phi}_{f}$

Grouped forcing eigenvectors for the positive and negative going elastic waves at frequency ha

$\gamma$

Displacement and forcing eigenvectors

Propagation constant and eigenvalue of the wave propagation eigenproblem

$\tau$

Generalised time variable

$\omega$

Fundamental input angular frequency 
aircraft's availability and significant financial losses for the operator. The online nondestructive detection and evaluation of damage in industrial structural components is of paramount importance for monitoring the condition and residual life estimation of in-service structures. Linear ultrasonic Guided Wave (GW) techniques have been widely employed for this purpose. These techniques however are primarily sensitive to gross defects but much less sensitive to micro-damage. Nonlinear acousto-ultrasonic techniques, have been steadily receiving increasing attention during the last decade. Complex wave phenomena such as higher and subharmonic wave generation, nonlinear resonances or mixed frequency response can be induced by the two principal sources of nonlinearity in the structural system, namely nonlinear elasticity and contact nonlinearity.

Elastic wave distortion and generation of higher harmonics during propagation in nonlinear media has been reported as early as in [3]. The first attempt for modelling wave interaction with nonlinear joints can be found in [4, 5]. It has been widely demonstrated that nonlinear ultrasonic techniques can be successfully deployed for detecting cracks as well as distributed structural deterioration (e.g. fatigue) $[6,7,8,9,10,11,12]$. The success of the developed methods is based on predicting and measuring the nonlinearities-induced wave effects which are pronounced in damaged and degraded structures but nearly unmeasurable in the undamaged ones. A number of Nonlinear Elastic Wave Spectroscopy (NEWS) approaches $[13,14,15]$ have also been presented and proved capable of detecting the presence of damage of very small sizes (in the order of $0.1 \mathrm{~mm}$ ) in composite structures. Wave propagation and material degradation detection in 1-D and 2-D media was investigated through a spring model in [16, 17]. A numerical scheme for predicting nonlinear wave interaction with an interface of rough surfaces in contact was presented in [18]. The non-collinear mixing of bulk shear waves investigated in [19] presented significant potential for assessing material state than other nonlinear ultrasonic techniques because system nonlinearities can be both independently measured and largely eliminated. The development of an analytical framework for modelling the multi-modal guided wave interaction with damage was presented by the authors of [20, 21]. In [22], a numerical scheme was presented in order to quantify the amplitude of the reflected compression and Rayleigh waves when impinging at the edge of an elastic plate. In [23] the authors coupled linear to nonlinear FE segments and investigated wave interaction with damage in 3-D solid media by means of a Landau's theory. Detection of fatigue damage in composite structures through higher harmonics generations has also recently been reported [24]. The short reviews provided by the authors in [25, 26] are informative on the general progress of nonlinear ultrasonics, while a comprehensive outline on the techniques dedicated to predicting and measuring higher harmonic generation in metallic structures is presented in [27]. An inclusive review on modelling wave-crack nonlinear interaction phenomena can be found in [28]. Despite the aforementioned attempts to capture wave interaction with LSNs, there is currently no generic computational scheme for predicting these quantities for composite layered structures.

The FE based wave propagation analysis within periodic structures was firstly considered in [29]. The wave dispersion characteristics within the layered media can be accurately predicted for a very wide frequency range, by solving a polynomial eigenvalue problem for the propagation constants to be sought. The work was extended to 2-D media in [30]. The WFE method was introduced in [31] in order to facilitate the post-processing of the 
eigenproblem solutions and further improve the computational efficiency of the method. The vibration of a uniform waveguide through the WFE technique was investigated in $[32,33]$. The method was also employed in order to predict the dynamic and vibroacoustic response $[34,35,36]$ of layered structures. The same FE based approach has been employed in order to compute the reflection and transmission coefficients of waves impinging on linear joints of finite dimensions $[37,38]$.

In this work, a generic FE-based scheme for computing wave interaction with LSNs is presented for the first time. Guided wave reflection, transmission and conversion is computed through a wave and finite element approach. The scheme couples wave propagation properties within linear structural waveguides to a LN and is able to determine the generation higher and sub-harmonics for each wave type through a harmonic balance projection. The new approach can predict reflections and transmissions at harmonic frequencies with a speed that is orders of magnitude faster than conventional transient FE solutions. The structure can be of arbitrary complexity, layering and material characteristics as FE modelling is employed. A Galerkin projection is used to transform the system of nonlinear differential equations of motion into a set of nonlinear algebraic equations which is subsequently solved through a Newton's iteration method. The generation of wave harmonics, as well as amplitude-dependent wave reflection and transmission coefficients are reported through the exhibited numerical case studies. This is the first approach that can accurately and efficiently map the frequency-dependent interactions of guided waves with nonlinearities in complex structures.

The paper is organized as follows: In Sec.2 the formulation of the wave and finite element method for predicting acoustic and ultrasonic GW interaction with LSNs is presented. A description of the Galerkin projection as well as of the Newton's iteration scheme employed for solving the system of nonlinear differential equations is also given. In Sec.3 the proposed method is validated for two different waveguides through comparison to full transient FE analyses. Conclusions on the presented work are given in Sec.4.

\section{Elastic wave interaction with structural nonlinearities}

\subsection{Computing wave propagation in a layered structure through a wave and finite element method}

Elastic wave propagation is considered in the $x$ direction of the linear layered waveguide of Fig.1. The problem can be condensed using a transfer matrix approach as in [31]. The frequency dependent Dynamic Stiffness Matrix (DMS) of the waveguide's periodic segment can be partitioned with regard to its left/right sides and internal DoF as

$$
\left[\begin{array}{ccc}
\mathbf{D}_{L L} & \mathbf{D}_{L I} & \mathbf{D}_{L R} \\
\mathbf{D}_{I L} & \mathbf{D}_{I I} & \mathbf{D}_{I R} \\
\mathbf{D}_{R L} & \mathbf{D}_{R I} & \mathbf{D}_{R R}
\end{array}\right]\left\{\begin{array}{c}
\mathbf{q}_{L} \\
\mathbf{q}_{I} \\
\mathbf{q}_{R}
\end{array}\right\}=\left\{\begin{array}{c}
\mathbf{f}_{L} \\
\mathbf{0} \\
\mathbf{f}_{R}
\end{array}\right\}
$$

with $\mathbf{q}$ the displacement and $\mathbf{f}$ the forcing vectors. Using a dynamic condensation for the internal DoF the problem can be expressed as

$$
\left[\begin{array}{cc}
\mathbf{D}_{L L}-\mathbf{D}_{L I} \mathbf{D}_{I I}^{-1} \mathbf{D}_{I L} & \mathbf{D}_{L R}-\mathbf{D}_{L I} \mathbf{D}_{I I}^{-1} \mathbf{D}_{I R} \\
\mathbf{D}_{R L}-\mathbf{D}_{R I} \mathbf{D}_{I I}^{-1} \mathbf{D}_{I L} & \mathbf{D}_{R R}-\mathbf{D}_{R I} \mathbf{D}_{I I}^{-1} \mathbf{D}_{I R}
\end{array}\right]\left\{\begin{array}{c}
\mathbf{q}_{L} \\
\mathbf{q}_{R}
\end{array}\right\}=\left\{\begin{array}{c}
\mathbf{f}_{L} \\
\mathbf{f}_{R}
\end{array}\right\}
$$




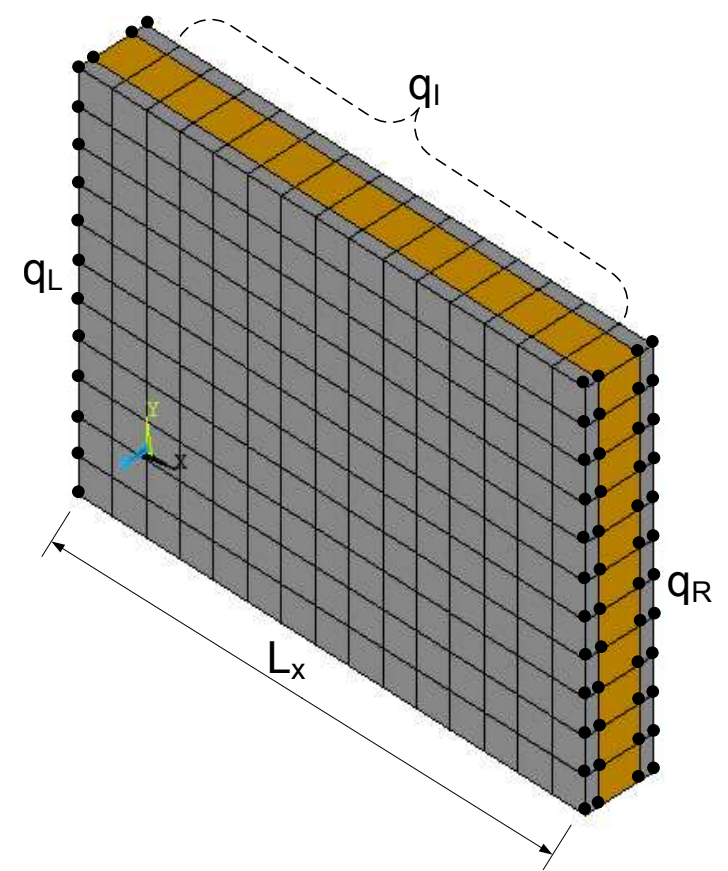

Figure 1: Caption of the WFE modelled composite waveguide with the left and right side nodes $\mathbf{q}_{L}, \mathbf{q}_{R}$ bullet marked. The range of interior nodes $\mathbf{q}_{I}$ is also illustrated.

Assuming that no external forces are applied on the segment the displacement continuity and force equilibrium equations at the interface of two consecutive periodic segments $s$ and $s+1$ give

$$
\begin{array}{r}
\mathbf{q}_{L}^{s+1}=\mathbf{q}_{R}^{s} \\
\mathbf{f}_{L}^{s+1}=-\mathbf{f}_{R}^{s}
\end{array}
$$

Using Eqs.2,3 the relation of the displacements and forces of the left and right sides of the segment can be written as

$$
\left\{\begin{array}{c}
\mathbf{q}_{L}^{s+1} \\
\mathbf{f}_{L}^{s+1}
\end{array}\right\}=\mathbf{T}\left\{\begin{array}{c}
\mathbf{q}_{L}^{s} \\
\mathbf{f}_{L}^{s}
\end{array}\right\}
$$

and the expression of the symplectic transfer matrix $\mathbf{T}$ can be formulated

$$
\mathbf{T}=\left[\begin{array}{ll}
\mathbf{D}_{11} & \mathbf{D}_{12} \\
\mathbf{D}_{21} & \mathbf{D}_{22}
\end{array}\right]_{[2 j \times 2 j]}
$$

with

$$
\begin{aligned}
\mathbf{D}_{11} & =-\left(\mathbf{D}_{L R}-\mathbf{D}_{L I} \mathbf{D}_{I I}^{-1} \mathbf{D}_{I R}\right)^{-1}\left(\mathbf{D}_{L L}-\mathbf{D}_{L I} \mathbf{D}_{I I}^{-1} \mathbf{D}_{I L}\right) \\
\mathbf{D}_{12}= & \left(\mathbf{D}_{L R}-\mathbf{D}_{L I} \mathbf{D}_{I I}^{-1} \mathbf{D}_{I R}\right)^{-1} \\
\mathbf{D}_{21}= & -\mathbf{D}_{R L}+\mathbf{D}_{R I} \mathbf{D}_{I I}^{-1} \mathbf{D}_{I L}+ \\
& +\left(\mathbf{D}_{R R}-\mathbf{D}_{R I} \mathbf{D}_{I I}^{-1} \mathbf{D}_{I R}\right)\left(\mathbf{D}_{L R}-\mathbf{D}_{L I} \mathbf{D}_{I I}^{-1} \mathbf{D}_{I R}\right)^{-1}\left(\mathbf{D}_{L L}-\mathbf{D}_{L I} \mathbf{D}_{I I}^{-1} \mathbf{D}_{I L}\right) \\
\mathbf{D}_{22}= & -\left(\mathbf{D}_{R R}-\mathbf{D}_{R I} \mathbf{D}_{I I}^{-1} \mathbf{D}_{I R}\right)\left(\mathbf{D}_{L R}-\mathbf{D}_{L I} \mathbf{D}_{I I}^{-1} \mathbf{D}_{I R}\right)^{-1}
\end{aligned}
$$


With a wave propagating freely along the $x$ direction, the propagation constant $\gamma=\mathrm{e}^{-\mathrm{i} k L_{x}}$ relates the right and left nodal displacements and forces by

$$
\begin{array}{r}
\mathbf{q}_{R}^{s}=\gamma \mathbf{q}_{L}^{s} \\
\mathbf{f}_{R}^{s}=-\gamma \mathbf{f}_{L}^{s}
\end{array}
$$

By substituting Eqs.3,7 in Eq.4, the free wave propagation is described by the eigenproblem

$$
\gamma\left\{\begin{array}{c}
\mathbf{q}_{L}^{s} \\
\mathbf{f}_{L}^{s}
\end{array}\right\}=\mathbf{T}\left\{\begin{array}{c}
\mathbf{q}_{L}^{s} \\
\mathbf{f}_{L}^{s}
\end{array}\right\}
$$

whose eigenvalues $\gamma_{w}$ and eigenvectors $\boldsymbol{\phi}_{w}=\left\{\begin{array}{c}\boldsymbol{\phi}_{q} \\ \boldsymbol{\phi}_{f}\end{array}\right\}$ solution sets provide a comprehensive description of the propagation constants and the wave mode shapes for each of the elastic waves propagating in the structural waveguide at a specified angular frequency $\omega$. Both positive going (with $\gamma_{w}^{+}$and $\left.\boldsymbol{\phi}_{w}^{+}\right)$and negative going waves $\left(\gamma_{w}^{-}\right.$and $\left.\boldsymbol{\phi}_{w}^{-}\right)$are sought through the eigensolution. Positive going waves are characterised [38] by

$$
\begin{gathered}
\left|\gamma_{w}^{+}\right| \leq 1, \\
\mathfrak{R}\left(\mathrm{i} \omega \boldsymbol{\phi}_{f}^{+\top} \boldsymbol{\phi}_{q}^{+}\right)<0 \text { if }\left|\gamma_{w}^{+}\right|=1
\end{gathered}
$$

stating that when a wave is travelling in the positive $x$ direction its amplitude should be decreasing, or that if its amplitude remains constant (in the case of propagating waves with complete absence of attenuation), then there is time averaged power transmission in the positive direction.

\subsection{Wave interaction with linear localised structural inhomogeneities}

The layered and periodic in the $x$ direction waveguide of Fig. 1 is hereby considered, with its propagation constants for the elastic waves travelling in the $x$ direction sought as described in Sec.2.1. For the sake of progressive presentation of the approach, we are initially assuming a system of two waveguides connected through a linear structural coupling element which is entirely FE modelled and which has different mechanical characteristics than the ones of the two waveguides coupled to it. A depiction of the system is presented in Fig.2. As already stated, each waveguide can be of different layering and can also support a different number $W_{n}$ of propagating waves at a given angular frequency $\omega$. An extensive description of how to deal with waveguides having different layouts and meshes can be found in [37]. Each supported wavemode $w$ with $w \in\left[1 \cdots W_{1}\right]$ for waveguide 1 in the system can be grouped as

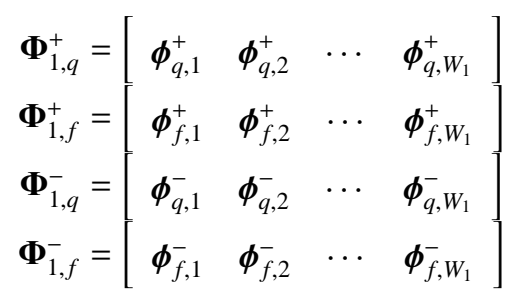

with each matrix being of dimension $\left[j \times W_{1}\right]$. Similarly the equivalent expressions for waveguide 2 (i.e. $\boldsymbol{\Phi}_{2, q}^{+}, \boldsymbol{\Phi}_{2, f}^{+}, \boldsymbol{\Phi}_{2, q}^{-}, \boldsymbol{\Phi}_{2, f}^{-}$) can be obtained with eigenvectors being normalised to unity. The wave modes of the entire system can be computed 


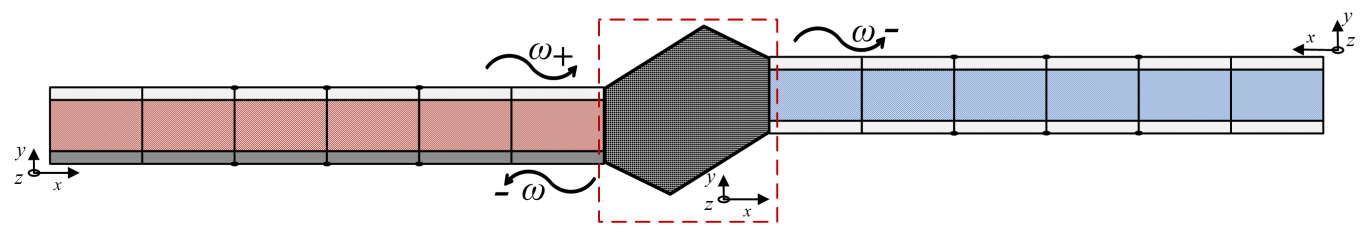

Figure 2: Periodic elastic waveguides connected through a coupling element exhibiting inhomogeneous but linear mechanical behaviour. Coupling element is depicted in the highlighted frame and is entirely FE modelled. A positive going wave ${ }^{+}$of angular frequency $\omega$ impinging on the coupling element will give rise to reflected and transmitted outgoing ${ }^{-}$waves of the same frequency.

for the fundamental excitation frequency $\omega$ and be grouped as

$$
\boldsymbol{\Phi}_{q}^{+}=\left[\begin{array}{cc}
\boldsymbol{\Phi}_{1, q}^{+} & \mathbf{0} \\
\mathbf{0} & \boldsymbol{\Phi}_{2, q}^{+}
\end{array}\right]
$$

with similar expressions standing for $\boldsymbol{\Phi}_{f}^{+}, \boldsymbol{\Phi}_{q}^{-}, \boldsymbol{\Phi}_{f}^{-}$. Assuming that the two waveguides have the same number of degrees of freedom $j$ at their interfaces and that the same number of waves is retained for both of them in the wave basis, then the size of the above table will be $[2 j \times 2 W]$. For each waveguide, the local coordinate system is defined such that the waveguide's axis is directed towards the joint (direction + ). The rotation matrix $\mathbf{R}_{n}$ transforms the DoFs from the local to the global coordinates of the system. For the two-waveguide system, rotation matrices can be grouped in a block diagonal matrix $\mathbf{R}$ as

$$
\mathbf{R}=\left[\begin{array}{cc}
\mathbf{R}_{1} & \mathbf{0} \\
\mathbf{0} & \mathbf{R}_{2}
\end{array}\right]_{[2 j \times 2 j]}
$$

The equation of motion for the linear, FE modelled coupling element can be in general written as

$$
\mathbb{M} \ddot{\mathbf{z}}(t)+\mathbb{C} \dot{\mathbf{z}}(t)+\mathbb{K} \mathbf{z}(t)=\mathbf{f}_{\text {ext }}(t)
$$

with $\mathbf{f}_{\text {ext }}(t)$ being the external forces applied to the coupling element by the two waveguides. The continuity conditions for the element give

$$
\mathbf{z}(t)=\mathbf{R q}(t)
$$

with $\mathbf{q}=\left[\mathbf{q}_{1}^{\top} \mathbf{q}_{2}^{\top}\right]_{[2 j \times 1]}^{\top}$. The equilibrium at the coupling element gives

$$
\mathbf{f}_{e x t}(t)-\mathbf{R f}(t)=\mathbf{0}
$$

with $\mathbf{f}(t)$ the set of forces applied by the impinging and outgoing waves to the coupling element.

\subsubsection{Calculation of the wave scattering matrix}

Waves of the fundamental excitation frequency $\omega$ having amplitudes $\mathbf{a}_{1}^{\omega,+}$ are impinging on the coupling element from the side of waveguide 1 . Their interaction with the coupling element will give rise to reflected waves of amplitudes $\mathbf{a}_{1}^{\omega,-}=\mathbf{r}_{1,1}^{\omega} \mathbf{a}_{1}^{\omega,+}$ in waveguide 1, while they also give rise to transmitted waves of amplitudes $\mathbf{a}_{2}^{\omega,-}=\mathbf{c}_{2,1}^{\omega} \mathbf{a}_{1}^{\omega,+}$ in 
the second waveguide with $\mathbf{r}_{1,1}^{\omega}$ and $\mathbf{c}_{2,1}^{\omega}$ being matrices containing the reflection and transmission coefficients of the coupling element at frequency $\omega$. These coefficients define the wave scattering matrix $\mathbf{S}^{\omega}$ of the joint, whose partitions relate the amplitudes of the incident and scattered waves as

$$
\mathbf{a}^{\omega,-}=\mathbf{S}^{\omega} \mathbf{a}^{\omega,+}
$$

with $\mathbf{a}_{[2 W \times 1]}^{\omega,+}$ the vector containing the amplitudes of the incoming waves moving towards the coupling element and $\mathbf{a}_{[2 W \times 1]}^{\omega,-}$ the vector containing the amplitudes of the reflected and transmitted outgoing waves. The wave scattering matrix $\mathbf{S}^{\omega}$ for the two-waveguide linear system can be written as

$$
\mathbf{S}^{\omega}=\left[\begin{array}{ccccc}
r_{1,1} & \cdots & c_{1, W} & \cdots & c_{1,2 W} \\
\cdots & \cdots & \cdots & \cdots & \cdots \\
c_{W, 1} & \cdots & r_{W, W} & \cdots & c_{W, 2 W} \\
\cdots & \cdots & \cdots & \cdots & \cdots \\
c_{2 W, 1} & \cdots & c_{2 W, W} & \cdots & r_{2 W, 2 W}
\end{array}\right]_{[2 W \times 2 W]}
$$

which can be computed for the selected range of harmonics of the fundamental frequency $\omega$. The motion of the waveguides in the physical 3D coordinate system is described in terms of displacements $\mathbf{q}$ and forces $\mathbf{f}$. On the other hand, in the wave domain the same motion can be described as a linear superposition of the retained propagating wave vectors $\boldsymbol{\Phi}_{n, q}^{\omega,+-}, \boldsymbol{\Phi}_{n, f}^{\omega,+-}$ along with their amplitudes $\mathbf{a}^{\omega,+}$ and $\mathbf{a}^{\omega,-}$. This superposition can be expressed as

$$
\begin{aligned}
\mathbf{q}_{n}(t) & =\boldsymbol{\Phi}_{n, q}^{\omega,+} \mathbf{a}_{n}^{\omega,+} \cos (\omega t)+\boldsymbol{\Phi}_{n, q}^{\omega,-} \mathbf{a}_{n}^{\omega,-} \cos (\omega t) \\
\mathbf{f}_{n}(t) & =\boldsymbol{\Phi}_{n, f}^{\omega,+} \mathbf{a}_{n}^{\omega,+} \cos (\omega t)+\boldsymbol{\Phi}_{n, f}^{\omega,-} \mathbf{a}_{n}^{\omega,-} \cos (\omega t)
\end{aligned}
$$

and by concatenating the corresponding vectors and matrices the general expressions for $\mathbf{q}$ and $\mathbf{f}$ for the system of waveguides can be expressed as

$$
\begin{aligned}
\mathbf{q}(t) & =\boldsymbol{\Phi}_{q}^{\omega,+} \mathbf{a}^{\omega,+} \cos (\omega t)+\boldsymbol{\Phi}_{q}^{\omega,-} \mathbf{a}^{\omega,-} \cos (\omega t) \\
\mathbf{f}(t) & =\boldsymbol{\Phi}_{f}^{\omega,+} \mathbf{a}^{\omega,+} \cos (\omega t)+\boldsymbol{\Phi}_{f}^{\omega,-} \mathbf{a}^{\omega,-} \cos (\omega t)
\end{aligned}
$$

It is noted that sin terms are not included in the above expansions as the phase of each wave scattering coefficient is captured by the imaginary part of the sought interaction coefficients $\mathbf{a}^{\omega,-}$. By performing the convenient substitution $\tau=\omega t$ and grouping the trigonometric terms the following expression can be acquired

$$
\mathbf{q}(\tau)=\boldsymbol{\Phi}_{q}^{\omega,+} \mathbf{T}_{2} \mathbf{a}^{\omega,+}+\mathbf{T}_{1} \mathcal{A}_{q}^{\omega,-}
$$

with $\mathbf{T}_{2}(\tau)=\left[\begin{array}{cc}\cos \tau & 0 \\ 0 & \cos \tau\end{array}\right], \mathbf{T}_{1}(\tau)=[\operatorname{diag}(\cos \tau)]_{[2 j \times 2 j]}$ and $\mathcal{A}_{q}^{\omega,-}$ being the generalised displacement wave interaction coefficient vector written as

$$
\mathcal{A}_{q}^{\omega,-}=\left\{\boldsymbol{\Phi}_{q}^{\omega,-} \mathbf{S}^{\omega} \mathbf{a}^{\omega,+}\right\}_{[2 j \times 1]}
$$


The following expressions can be immediately derived

$$
\begin{gathered}
\mathbf{f}(\tau)=\boldsymbol{\Phi}_{f}^{\omega,+} \mathbf{T}_{2} \mathbf{a}^{\omega,+}+\mathbf{T}_{1} \mathcal{A}_{f}^{\omega,-} \\
\dot{\mathbf{q}}(\tau)=\omega \boldsymbol{\Phi}_{q}^{\omega,+} \frac{\mathrm{d} \mathbf{T}_{2}}{\mathrm{~d} \tau} \mathbf{a}^{\omega,+}+\omega \frac{\mathrm{d} \mathbf{T}_{1}}{\mathrm{~d} \tau} \mathcal{A}_{q}^{\omega,-} \\
\ddot{\mathbf{q}}(\tau)=\omega^{2} \boldsymbol{\Phi}_{q}^{\omega,+} \frac{\mathrm{d}^{2} \mathbf{T}_{2}}{\mathrm{~d} \tau^{2}} \mathbf{a}^{\omega,+}+\omega^{2} \frac{\mathrm{d}^{2} \mathbf{T}_{1}}{\mathrm{~d} \tau^{2}} \mathcal{A}_{q}^{\omega,-}
\end{gathered}
$$

with $\mathcal{A}_{f}^{\omega,-}$ formulated similarly to $\mathcal{A}_{q}^{\omega,-}$. Substituting Eqs.20,22 into Eqs.14,15 and then into Eq.13 gives the generalised expression for the equation of motion of the coupling element

$$
\begin{gathered}
\omega^{2} \mathbb{M} \mathbf{R} \Phi_{q}^{\omega,+} \frac{\mathrm{d}^{2} \mathbf{T}_{2}}{\mathrm{~d} \tau^{2}} \mathbf{a}^{\omega,+}+\omega^{2} \mathbb{M} \mathbf{R} \frac{\mathrm{d}^{2} \mathbf{T}_{1}}{\mathrm{~d} \tau^{2}} \mathcal{A}_{q}^{\omega,-}+\omega \mathbb{C} \mathbf{R} \Phi_{q}^{\omega,+} \frac{\mathrm{d} \mathbf{T}_{2}}{\mathrm{~d} \tau} \mathbf{a}^{\omega,+}+ \\
+\omega \mathbb{C} \mathbf{R} \frac{\mathrm{d} \mathbf{T}_{1}}{\mathrm{~d} \tau} \mathcal{A}_{q}^{\omega,-}+\mathbb{K} \mathbf{R} \Phi_{q}^{\omega,+} \mathbf{T}_{2} \mathbf{a}^{\omega,+}+\mathbb{K} \mathbf{R} \mathbf{T}_{1} \mathcal{A}_{q}^{\omega,-}=\mathbf{R} \boldsymbol{\Phi}_{f}^{\omega,+} \mathbf{T}_{2} \mathbf{a}^{\omega,+}+\mathbf{R} \mathbf{T}_{1} \mathcal{A}_{f}^{\omega,-}
\end{gathered}
$$

A set of equations with the transmission and reflection coefficients as unknowns can be obtained through a Galerkin projection of Eq. 23 as

$$
\begin{aligned}
& \int_{0}^{2 \pi}\left[\mathbf{T}_{1}^{\top}\left[\omega^{2} \mathbb{M} \mathbf{R} \frac{\mathrm{d}^{2} \mathbf{T}_{1}}{\mathrm{~d} \tau^{2}}+\omega \mathbb{C} \mathbf{R} \frac{\mathrm{d} \mathbf{T}_{1}}{\mathrm{~d} \tau}+\mathbb{K} \mathbf{R} \mathbf{T}_{1}\right] \mathcal{A}_{q}^{\omega,-}-\mathbf{T}_{1}^{\top} \mathbf{R} \mathbf{T}_{1} \mathcal{A}_{f}^{\omega,-}\right] \mathrm{d} \tau+ \\
& \int_{0}^{2 \pi} \mathbf{T}_{1}^{\top}\left[\omega^{2} \mathbb{M} \mathbf{R} \boldsymbol{\Phi}_{q}^{\omega,+} \frac{\mathrm{d}^{2} \mathbf{T}_{2}}{\mathrm{~d} \tau^{2}}+\omega \mathbb{C} \mathbf{R} \boldsymbol{\Phi}_{q}^{\omega,+} \frac{\mathrm{d} \mathbf{T}_{2}}{\mathrm{~d} \tau}+\mathbb{K} \mathbf{R} \boldsymbol{\Phi}_{q}^{\omega,+} \mathbf{T}_{2}-\mathbf{R} \boldsymbol{\Phi}_{f}^{\omega,+} \mathbf{T}_{2}\right] \mathbf{a}^{\omega,+} \mathrm{d} \tau=\mathbf{0}
\end{aligned}
$$

A Newton's iterative scheme can be eventually employed in order to extract the wave interaction coefficients $\mathbf{S}^{\omega}$ out of Eq. 24 by exciting one by one the incoming waves for each waveguide (that is by setting all wave amplitudes in $\mathbf{a}^{\omega,+}$ to zero except for the investigated incoming wave). As no matrix inverse is involved for the computation of the wave interaction coefficients (in contrast to [38]), a reduced wave basis can be retained without ill-conditioning of the above expressions. It should be stressed that modelling non-conservative waveguides and coupling elements implies that all computed wavenumbers will be complex and the strict distinction between evanescent and propagating waves breaks down. In that case, an extended wave basis should be retained (all waves having a non-negligible real wavenumber part should be kept) in order to take into account for wave conversions induced by material damping. In most practical situations however, the assumption of a conservative coupling element yields reliable results for the wave interaction coefficients as discussed in [39].

\subsection{Wave interaction with structural nonlinearities}

It is hereby assumed that the modelled coupling element exhibits a specific and known nonlinear mechanical behaviour. It is still given that an incident wave of fundamental frequency $\omega$ is impinging on the coupling element, however this localised nonlinearity in the system will give rise to a number of waves of super-harmonic $(h \omega)$ and sub-harmonic $(\omega / h)$ frequencies during the interaction process (see Fig.3). The wave basis for each waveguide can be computed and grouped as in Eq.11, however in order to project wave motion in multiple sub-harmonics and superharmonics then the corresponding wave bases should also be included in the following calculations. The number 


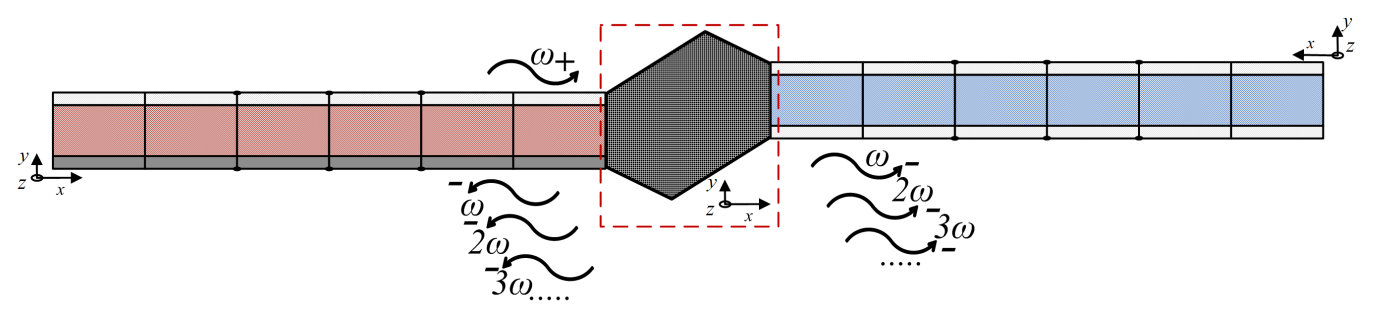

Figure 3: Periodic elastic waveguides connected through a coupling element exhibiting inelastic mechanical behaviour. A positive going wave ${ }^{+}$of angular frequency $\omega$ impinging on the coupling element will give rise to reflected and transmitted outgoing ${ }^{-}$waves of multiple subharmonic and superharmonic frequencies.

of higher harmonics $H$ and subharmonics $H_{m}$ to be computed and included in the wave basis depends on the order of nonlinearity of the modelled coupling element (see also [20,21]). Therefore each supported wavemode $w$ with $w \in\left[1 \cdots W_{n}\right]$ for each waveguide in the system has to be computed for the super and sub-harmonics $h \omega$ with $h \in\left[1 / H_{m} \cdots 1 \cdots H\right]$ and be grouped as

$$
\boldsymbol{\Phi}_{q}^{h \omega,+}=\left[\begin{array}{cc}
\boldsymbol{\Phi}_{1, q}^{h \omega,+} & \mathbf{0} \\
\mathbf{0} & \boldsymbol{\Phi}_{2, q}^{h \omega,+}
\end{array}\right]_{[2 j \times 2 W]}
$$

with similar expressions standing for $\boldsymbol{\Phi}_{f}^{h \omega,+}, \boldsymbol{\Phi}_{q}^{h \omega,-}, \boldsymbol{\Phi}_{f}^{h \omega,-}$. The equation of motion for the nonlinear, FE modelled coupling element can now be generically written as

$$
\mathbb{M} \ddot{\mathbf{z}}(t)+\mathbb{C} \dot{\mathbf{z}}(t)+\mathbb{K} \mathbf{z}(t)+\mathbf{f}_{N L}=\mathbf{f}_{\text {ext }}(t)
$$

with $\mathbf{f}_{N L}$ the nonlinear force vector induced by the coupling element's inherent inelastic behaviour. The same continuity and equilibrium conditions for the element are applied as expressed by Eqs.14,15. Waves of the fundamental excitation frequency $\omega$ having amplitudes $\mathbf{a}_{n}^{\omega,+}$ are impinging on the coupling element from waveguide 1 . For each considered harmonic $h \omega$, they give rise to reflected waves of amplitudes $\mathbf{a}_{1}^{h \omega,-}=\mathbf{r}_{1,1}^{h \omega} \mathbf{a}_{1}^{\omega,+}$ in the first waveguide, while they also generate transmitted waves of amplitudes $\mathbf{a}_{2}^{h \omega,-}=\mathbf{c}_{2,1}^{h \omega} \mathbf{a}_{1}^{\omega,+}$ in the second waveguide with $\mathbf{r}_{1,1}^{h \omega}$ and $\mathbf{c}_{2,1}^{h \omega}$ being matrices containing the reflection and transmission coefficients of the coupling element at each harmonic $h \omega$. These define the wave scattering matrix $\mathbf{S}^{h \omega}$ of the joint

$$
\mathbf{a}^{h \omega,-}=\mathbf{S}^{h \omega} \mathbf{a}^{\omega,+}
$$

which now has to be computed for each considered sub-harmonic and super-harmonic frequency. The wave scattering matrix $\mathbf{S}^{h \omega}$ now projects the incoming waves $\mathbf{a}^{\omega,+}$ of fundamental frequency $\omega$ to each of the higher and sub-harmonics $h \omega$. The matrix can be computed for the selected range of harmonics of the fundamental frequency $\omega$. Similarly to Eq.18, a superposition expression can be written for the case where wave energy escapes towards higher and subharmonics as

$$
\begin{aligned}
& \mathbf{q}_{n}(t)=\boldsymbol{\Phi}_{n, q}^{\omega,+} \mathbf{a}_{n}^{\omega,+} \cos (\omega t)+\sum_{h=1}^{H} \boldsymbol{\Phi}_{n, q}^{h \omega,-} \mathbf{a}_{n}^{h \omega,-} \cos (h \omega t)+\sum_{h=2}^{H_{m}} \mathbf{\Phi}_{n, q}^{h \omega,-} \mathbf{a}_{n}^{h \omega,-} \cos \left(\frac{1}{h} \omega t\right) \\
& \mathbf{f}_{n}(t)=\boldsymbol{\Phi}_{n, f}^{\omega,+} \mathbf{a}_{n}^{\omega,+} \cos (\omega t)+\sum_{h=1}^{H} \boldsymbol{\Phi}_{n, f}^{h \omega,-} \mathbf{a}_{n}^{h \omega,-} \cos (h \omega t)+\sum_{h=2}^{H_{m}} \boldsymbol{\Phi}_{n, f}^{h \omega,-} \mathbf{a}_{n}^{h \omega,-} \cos \left(\frac{1}{h} \omega t\right)
\end{aligned}
$$


and by concatenating the corresponding vectors and matrices the general expressions for $\mathbf{q}$ and $\mathbf{f}$ for the system of waveguides can be expressed as

$$
\begin{aligned}
& \mathbf{q}(t)=\boldsymbol{\Phi}_{q}^{\omega,+} \mathbf{a}^{\omega,+} \cos (\omega t)+\sum_{h=1}^{H} \boldsymbol{\Phi}_{q}^{h \omega,-} \mathbf{a}^{h \omega,-} \cos (h \omega t)+\sum_{h=2}^{H_{m}} \boldsymbol{\Phi}_{q}^{h \omega,-} \mathbf{a}^{h \omega,-} \cos \left(\frac{1}{h} \omega t\right) \\
& \mathbf{f}(t)=\boldsymbol{\Phi}_{f}^{\omega,+} \mathbf{a}^{\omega,+} \cos (\omega t)+\sum_{h=1}^{H} \boldsymbol{\Phi}_{f}^{h \omega,-} \mathbf{a}^{h \omega,-} \cos (h \omega t)+\sum_{h=2}^{H_{m}} \boldsymbol{\Phi}_{f}^{h \omega,-} \mathbf{a}^{h \omega,-} \cos \left(\frac{1}{h} \omega t\right)
\end{aligned}
$$

The above expressions can be employed to perform a cyclostationary projection of the behaviour of the system through the Harmonic Balance Method (HBM) [40]. By performing the convenient substitution $\tau=\omega t$ once again and grouping the trigonometric terms the following expression can be acquired

$$
\mathbf{q}(\tau)=\boldsymbol{\Phi}_{q}^{\omega,+} \mathbf{T}_{2} \mathbf{a}^{+}+\mathbf{T}_{1} \mathcal{A}_{q}^{\omega,-}
$$

with $\mathbf{T}_{2}(\tau)$ expressed as in Sec.2.2 and $\mathbf{T}_{1}(\tau)=\left[\operatorname{diag}(\cos \tau)_{[2 j \times 2 j]}, \operatorname{diag}(\cos 2 \tau)_{[2 j \times 2 j]}, \cdots, \operatorname{diag}(\cos H \tau)_{[2 j \times 2 j]}\right]_{[2 j \times 2 j H]}$, while $\mathcal{A}_{q}^{\omega,-}$ is the generalised displacement wave interaction coefficient vector now written as

$$
\mathcal{A}_{q}^{\omega,-}=\left\{\begin{array}{c}
\boldsymbol{\Phi}_{q}^{\omega,-} \mathbf{S}^{\omega} \mathbf{a}^{\omega,+} \\
\boldsymbol{\Phi}_{q}^{2 \omega,-} \mathbf{S}^{2 \omega} \mathbf{a}^{\omega,+} \\
\cdots \\
\boldsymbol{\Phi}_{q}^{H \omega,-} \mathbf{S}^{H \omega} \mathbf{a}^{\omega,+} \\
\cdots \\
\boldsymbol{\Phi}_{q}^{\frac{1}{H_{m}} \omega,-} \mathbf{S}^{\frac{1}{H_{m}} \omega} \mathbf{a}^{\omega,+}
\end{array}\right\}_{[2 j H \times 1]}
$$

The expressions of Eq.22 are still valid and Eq.23 for the generalised equation of motion of the coupling element is now modified as

$$
\begin{gathered}
\omega^{2} \mathbb{M} \mathbf{R} \boldsymbol{\Phi}_{q}^{\omega,+} \frac{\mathrm{d}^{2} \mathbf{T}_{2}}{\mathrm{~d} \tau^{2}} \mathbf{a}^{\omega,+}+\omega^{2} \mathbb{M} \mathbf{R} \frac{\mathrm{d}^{2} \mathbf{T}_{1}}{\mathrm{~d} \tau^{2}} \mathcal{A}_{q}^{\omega,-}+\omega \mathbb{C} \mathbf{R} \Phi_{q}^{\omega,+} \frac{\mathrm{d} \mathbf{T}_{2}}{\mathrm{~d} \tau} \mathbf{a}^{\omega,+}+ \\
+\omega \mathbb{C} \mathbf{R} \frac{\mathrm{d} \mathbf{T}_{1}}{\mathrm{~d} \tau} \mathcal{A}_{q}^{\omega,-}+\mathbb{K} \mathbf{R} \boldsymbol{\Phi}_{q}^{\omega,+} \mathbf{T}_{2} \mathbf{a}^{\omega,+}+\mathbb{K} \mathbf{R} \mathbf{T}_{1} \mathcal{A}_{q}^{\omega,-}+\mathbf{f}_{N L}=\mathbf{R} \boldsymbol{\Phi}_{f}^{\omega,+} \mathbf{T}_{2} \mathbf{a}^{(\omega,+}+\mathbf{R} \mathbf{T}_{1} \mathcal{A}_{f}^{\omega,-}
\end{gathered}
$$

It is noted that $\mathbb{K}$ in the above expression represents the elastic part of the mechanical behaviour of the coupling element. A set of nonlinear, algebraic equations can be obtained through a Galerkin projection [41] of Eq.32 back onto the set of harmonic solutions as

$$
\begin{aligned}
& \int_{0}^{2 H_{m} \pi}\left[\mathbf{T}_{1}^{\top}\left[\omega^{2} \mathbb{M} \mathbf{R} \frac{\mathrm{d}^{2} \mathbf{T}_{1}}{\mathrm{~d} \tau^{2}}+\omega \mathbb{C} \mathbf{R} \frac{\mathrm{d} \mathbf{T}_{1}}{\mathrm{~d} \tau}+\mathbb{K} \mathbf{R} \mathbf{T}_{1}\right] \mathcal{A}_{q}^{\omega,-}-\mathbf{T}_{1}^{\top} \mathbf{R} \mathbf{T}_{1} \mathcal{A}_{f}^{\omega,-}\right] \mathrm{d} \tau+ \\
& \int_{0}^{2 H_{m} \pi} \mathbf{T}_{1}^{\top}\left[\omega^{2} \mathbb{M} \mathbf{R} \mathbf{\Phi}_{q}^{\omega,+} \frac{\mathrm{d}^{2} \mathbf{T}_{2}}{\mathrm{~d} \tau^{2}}+\omega \mathbb{C} \mathbf{R} \boldsymbol{\Phi}_{q}^{\omega,+} \frac{\mathrm{d} \mathbf{T}_{2}}{\mathrm{~d} \tau}+\mathbb{K} \mathbf{R} \boldsymbol{\Phi}_{q}^{\omega,+} \mathbf{T}_{2}-\mathbf{R} \boldsymbol{\Phi}_{f}^{\omega,+} \mathbf{T}_{2}\right] \mathbf{a}^{\omega,+} \mathrm{d} \tau+ \\
& \int_{0}^{2 H_{m} \pi} \mathbf{T}_{1}^{\top} \mathbf{f}_{N L} \mathrm{~d} \tau=\mathbf{0}
\end{aligned}
$$

A Newton's iterative scheme similar to the one employed in Sec.2.2 can be used to extract the wave interaction coefficients $\mathbf{S}^{\omega / H_{m}}, \cdots \mathbf{S}^{\omega}, \mathbf{S}^{2 \omega}, \cdots \mathbf{S}^{H \omega}$ out of Eq.33 by exciting one by one the incoming waves for each waveguide. The generic iterative procedure of the employed Newton solution scheme is presented in Algorithm 1. 


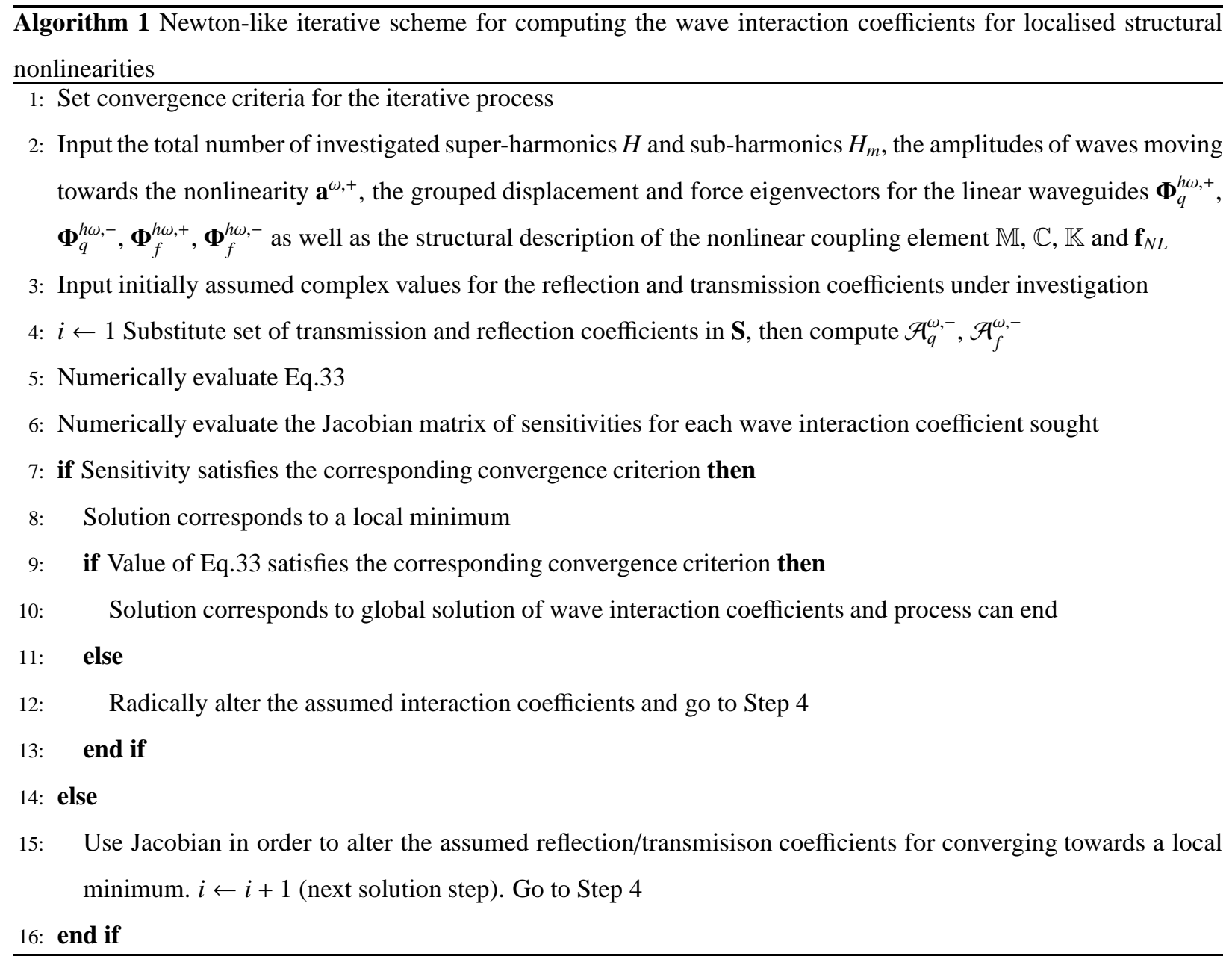




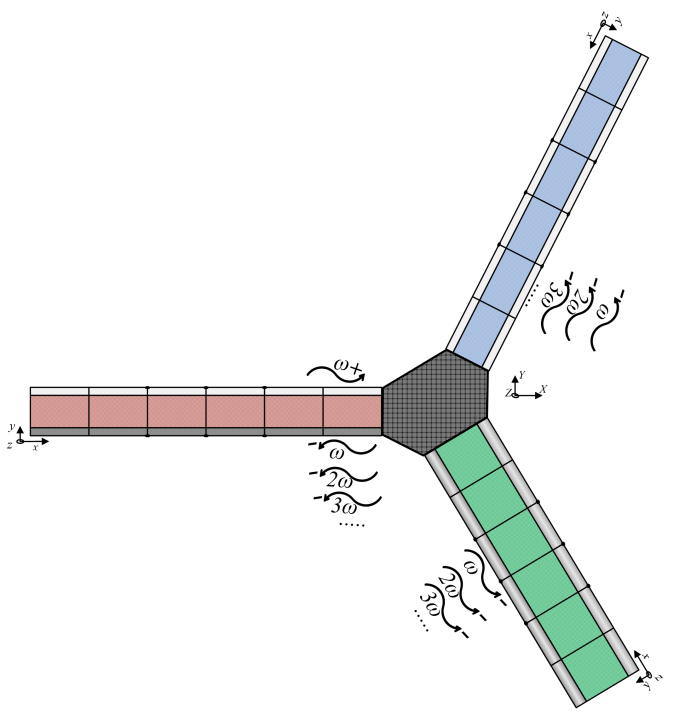

Figure 4: Periodic elastic waveguides connected through a coupling element exhibiting inelastic mechanical behaviour. A positive going wave ${ }^{+}$of angular frequency $\omega$ impinging on the coupling element will give rise to reflected and transmitted outgoing ${ }^{-}$waves of multiple subharmonic and superharmonic frequencies.

\subsection{Generalization to an arbitrary number of connected waveguides}

The scheme presented in Sec.2.3 can be generalised to include more than two waveguides connected at the nonlinear coupling element. In the general case, we are assuming a system of $N$ waveguides as in Fig.4. As already stated, each waveguide can be of different and arbitrary layering and can also support a different number $W_{n}$ of propagating waves at a given frequency. Each supported wavemode $w$ with $w \in\left[1 \cdots W_{n}\right]$ for the $n$th waveguide in the system can be grouped as

$$
\begin{aligned}
\boldsymbol{\Phi}_{n, q}^{h \omega,+} & =\left[\begin{array}{llll}
\boldsymbol{\phi}_{q, 1}^{h \omega,+} & \boldsymbol{\phi}_{q, 2}^{h \omega,+} & \cdots & \boldsymbol{\phi}_{q, W}^{h \omega,+}
\end{array}\right] \\
\boldsymbol{\Phi}_{n, f}^{h \omega,+} & =\left[\begin{array}{llll}
\boldsymbol{\phi}_{f, 1}^{h \omega,+} & \boldsymbol{\phi}_{f, 2}^{h \omega,+} & \cdots & \boldsymbol{\phi}_{f, W}^{h \omega,+}
\end{array}\right] \\
\boldsymbol{\Phi}_{n, q}^{h \omega,-} & =\left[\begin{array}{llll}
\boldsymbol{\phi}_{q, 1}^{h \omega,-} & \boldsymbol{\phi}_{q, 2}^{h \omega,-} & \cdots & \boldsymbol{\phi}_{q, W}^{h \omega,-}
\end{array}\right] \\
\boldsymbol{\Phi}_{n, f}^{h \omega,-} & =\left[\begin{array}{llll}
\boldsymbol{\phi}_{f, 1}^{h \omega,-} & \boldsymbol{\phi}_{f, 2}^{h \omega,-} & \cdots & \boldsymbol{\phi}_{f, W}^{h \omega,-}
\end{array}\right]
\end{aligned}
$$

with each matrix being of dimension $\left[j \times W_{n}\right]$. The wavemodes of the entire system can be computed for each waveguide $n$ with $n \in[1 \cdots N]$ and for the fundamental excitation frequency $\omega$ as well as for the higher and subharmonics $h \omega$ with $h \in\left[1 / H_{m} \cdots 1 \cdots H\right]$ and be grouped as

$$
\boldsymbol{\Phi}_{q}^{h \omega,+}=\left[\begin{array}{cccc}
\boldsymbol{\Phi}_{1, q}^{h \omega,+} & \mathbf{0} & \ldots & \mathbf{0} \\
\mathbf{0} & \boldsymbol{\Phi}_{2, q}^{h \omega,+} & \ldots & \mathbf{0} \\
\ldots & \ldots & \ldots & \ldots \\
\mathbf{0} & \mathbf{0} & \cdots & \boldsymbol{\Phi}_{N, q}^{h \omega,+}
\end{array}\right]_{[j N \times W N]}
$$


with similar expressions standing for $\boldsymbol{\Phi}_{f}^{h \omega,+}, \boldsymbol{\Phi}_{q}^{h \omega,-}, \boldsymbol{\Phi}_{f}^{h \omega,-}$. For each waveguide, the local coordinate system is defined such that the waveguide's axis is directed towards the joint (direction + ). The rotation matrix $\mathbf{R}_{n}$ transforms the DoFs from the local to the global coordinates of the system. Rotation matrices can be grouped in a block diagonal matrix $\mathbf{R}$ as

$$
\mathbf{R}=\left[\begin{array}{cccc}
\mathbf{R}_{1} & \mathbf{0} & \cdots & \mathbf{0} \\
\mathbf{0} & \mathbf{R}_{2} & \cdots & \mathbf{0} \\
\cdots & \cdots & \cdots & \cdots \\
\mathbf{0} & \mathbf{0} & \cdots & \mathbf{R}_{N}
\end{array}\right]_{[j N \times j N]}
$$

The procedure then follows the same steps through Eq.26 to Eq.33 in order to sought the set of transmission and reflection coefficients for each harmonic frequency and each modelled waveguide when a single wave type is impinging on the coupling element. The increase in the number of waveguides and the size of the concatenated wave basis can radically increase the computational burden of the iterative solution scheme. Parallel computing algorithms can be employed as the most straightforward tool to minimise the computational cost.

\section{Numerical case studies}

\subsection{Validation of wave interaction coefficients through full FE simulations}

The above exhibited scheme will be validated through full FE transient simulations. The entire 1D structure is modelled through linear, brick solid FE and the displacements $\boldsymbol{\phi}_{q, w}^{\omega,+}$ corresponding to a certain propagating wave mode $w$ are imposed on one of its ends $\chi_{0}$ (see also Fig. 5 for illustration). A Hanning-windowed signal containing 11 cycles is selected in order to minimise spectral leakage for the propagating packet. The displacements at a certain monitoring cross-section of the waveguide laying before the LSN at distance $\chi_{1}$, are recorded along with the incident and reflected wave signatures. The reflection coefficient is defined as the ratio of energies of the reflected signal over the incident one. These wave packet energies can effectively be computed through a Fourier transform over the time ranges corresponding to wave incidence and reflection. The same transform can directly furnish the wave energies at each generated sub-harmonic and super-harmonic. Transmission coefficients can be computed through the same approach with the monitored cross-section laying after the LSN.

Spatial (element size) and temporal (integration time step) resolutions of the finite element model are chosen to ensure solution convergence while ensuring the model computational size is reasonable. Time step is selected equal to $\frac{1}{20 f_{\max }}$ with $f_{\max }$ the maximum harmonic frequency considered in the problem. The element size is chosen equal to $\frac{\lambda_{\min }}{20}$ where $\lambda_{\min }$ is the minimum wavelength to be taken into account in the wave basis (estimated through the computed wavenumbers from the wave and finite element scheme in Sec.2.1). This discretisation is adequate to avoid spatial aliasing and ensure the inclusion of higher harmonics [42]. The smaller the integration time step $\mathrm{d} t$, the better the accuracy of the numerical result which however induces a greater computational cost. 


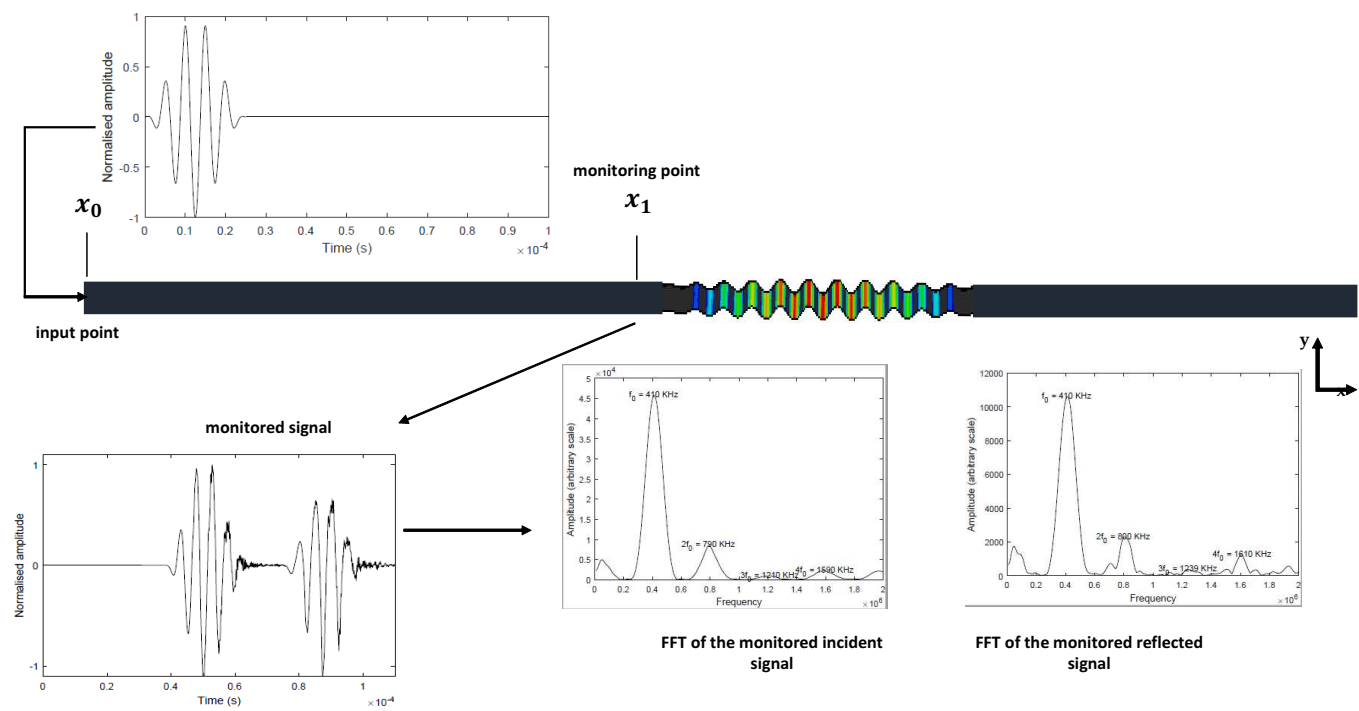

Figure 5: Schematic representation of the computation of reflection/transmission coefficients through a transient FE analysis. A Hanning windowed signal is imposed on one end of the waveguide and the response is measured at monitoring cross-section $\chi_{1}$. The energy of each wave packet is then calculated through a Fourier transform.

\subsubsection{Projecting structural motion of a waveguide on its wave basis}

The full FE simulations provide a complete description of the global displacements of a waveguide's cross-section as a function of time. In certain cases however wave conversion may take place at the point of the LSN. It is therefore essential to decompose these global displacements into a sum of independent wave mode displacements using the wave superposition principle. It is indeed helpful to note that once the wave displacement basis

$$
\boldsymbol{\Phi}_{n, q}^{+}=\left[\begin{array}{llll}
\boldsymbol{\phi}_{q, 1}^{+} & \boldsymbol{\phi}_{q, 2}^{+} & \cdots & \boldsymbol{\phi}_{q, W}^{+}
\end{array}\right]
$$

for a certain composite waveguide has been determined through standard WFE computations, then any motion within the structure can be described as a superposition of these wave mode shapes as

$$
\mathbf{q}_{n}^{+}=\boldsymbol{\Phi}_{n, q}^{\omega,+} \mathbf{a}^{\omega,+}
$$

with $\mathbf{a}^{\omega,+}$ the vector of amplitude coefficients denoting the participation of each wave type in the global waveguide motion and which can be obtained for each cross-section and each instant in time if the physical displacements $\mathbf{q}_{n}^{+}$ are known through inverting the above expression (a pseudoinverse can be employed when a reduced wave basis is kept and $\boldsymbol{\Phi}_{n, q}^{\omega,+}$ is not square). By registering $\mathbf{a}^{\omega,+}$ as a function of time for a certain cross-section of the waveguide and employing a Fourier transform, the frequency content for each wave type and subsequently the reflection and transmission coefficients for each investigated wave motion is straightforward to obtain through the results of a full FE transient simulation. 


\subsection{Validation for an aluminium beam}

The computational scheme exhibited above is initially applied in a discretized aluminium beam system as presented in Fig.6. The configuration comprises two waveguides having a cross-section of $8 \mathrm{~mm} \times 12 \mathrm{~mm}$. The two waveguides can in general have different characteristics however in this case they are both assumed to be made of aluminium and are connected through a nonlinear element governed by a third order nonlinearity implemented within the coupling element. A damping loss factor equal to $\eta=1 \%$ was considered. By modelling the identical waveguides through the WFE approach presented in Sec.2.1, it can be found that four waves can propagate within the structure.

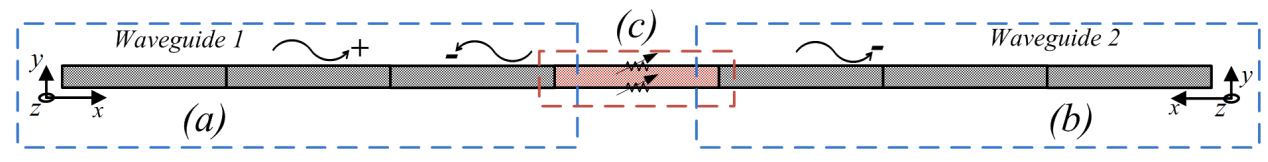

Figure 6: Schematic representation of the two healthy and elastic monolayer waveguides (a) and (b) coupled through a nonlinear element (c).

In order to validate the presented approach in the linear domain first, the results derived through Eq.24 are initially compared against the methodology presented in [38] when a purely elastic coupling element is considered connecting the two waveguides with $E_{c e}=E_{w g} / 2$ where $E_{c e}$ stands for the Young's modulus of the coupling element and $E_{w g}$ for the one of the waveguides. The results for this linearly softened coupling element using both approaches are presented in Fig.7.

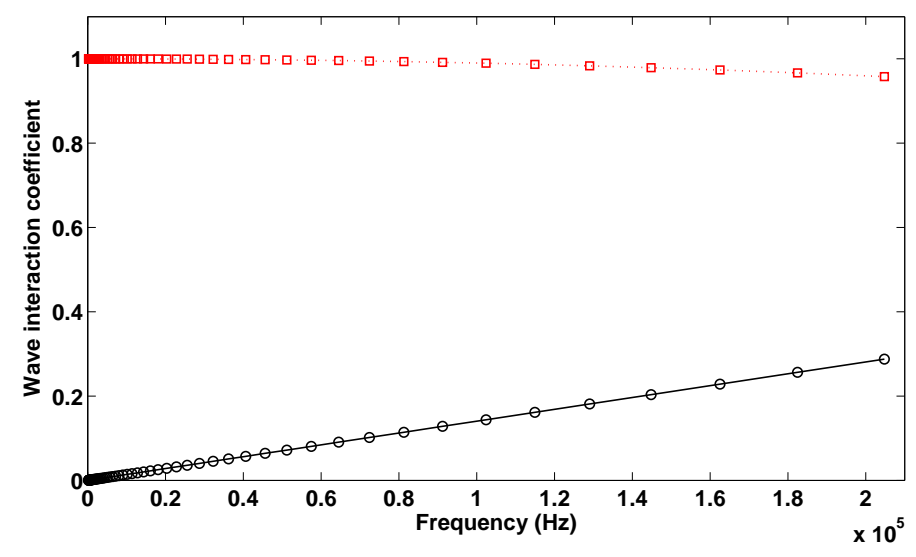

Figure 7: Absolute values of the wave interaction coefficients for a linearly softened coupling element connecting the two waveguides: Reflection coefficients for the pressure wave computed according to the current scheme (-), Reflection coefficients computed as in [38] (o), Transmission coefficients computed according to the current scheme $(\cdots)$, Transmission coefficients computed as in [38] ( $\square$ ).

Excellent agreement is observed for the reflection and transmission coefficients, while the interaction coefficients for the higher and sub-harmonic outgoing waves are as expected null. A nonlinear coupling element is subsequently considered comprising a variable stiffness $k_{n l}$ being dependent on the instantaneous deformation distance $d$. The imposed amplitude of the incoming propagating wave is equal to $10 \mu \mathrm{m}$. The results on the harmonic reflections for a nonlinearly hardening element (computed through Eq.33) are presented below in Figs.8, 9. As expected and 
demonstrated in Fig.9, the LSN gives rise to higher harmonic waves which are generally more pronounced at higher excitation frequencies.

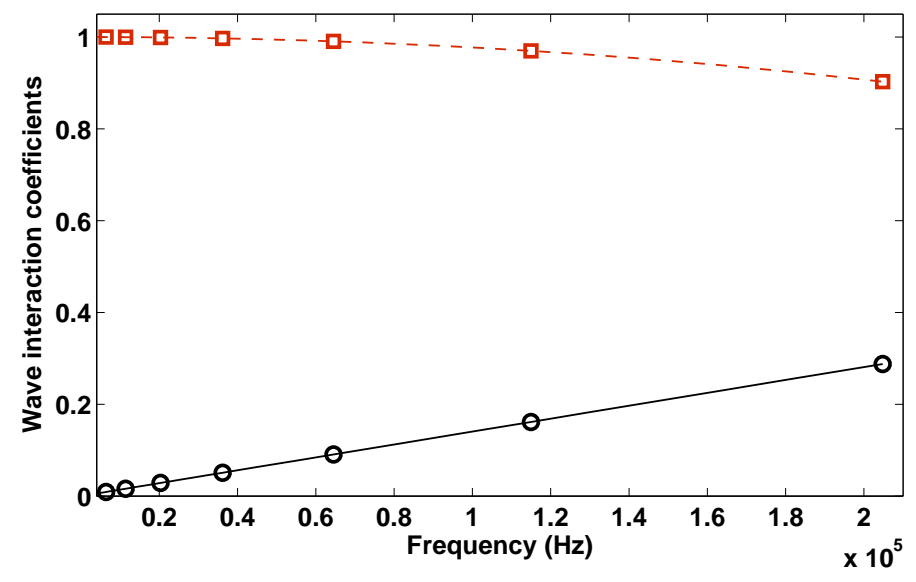

Figure 8: Absolute values of the wave reflection and transmission coefficients of the pressure propagating wave at frequency $\omega$ for a nonlinearly hardened element with $k_{n l}=1 \mathrm{e} 13 \mathrm{~N} / \mathrm{m}^{3}$ : Reflection coefficients computed according to the current scheme (-), Reflection coefficients computed through a full transient FE calculation (o), Transmission coefficients computed according to the current scheme (-,), Transmission coefficients computed through a full transient FE calculation ( $\square$ ).

It is initially observed that an excellent agreement exists between the presented approach and the full FE transient solution performed using ANSYS ${ }^{\circledR}$, especially for reflections at the excitation frequency $\omega$. Linear, solid brick ANSYS ${ }^{\circledR}$ elements were used for the periodic unit cell, as well as for the full FE model. The exhibited scheme was programmed using the R2013a version of MATLAB ${ }^{\circledR}$. Despite the fact that MATLAB ${ }^{\circledR}$ solving capabilities are far from being optimal, the computational time was reduced by a factor of 11.5 (3350 seconds/frequency for a full FE computation to 290 seconds for the presented scheme). This computational time reduction owed to the employment of periodic structure theory will be significantly greater for larger and more complex structural models as well as for higher frequencies (in the $\mathrm{MHz}$ range) when a much finer mesh will be needed for simulating wave propagation.

\subsection{Validation for a layered composite beam}

The presented approach is next validated for an asymmetric layered composite beam with aluminium facesheets and a polyurethane core having a cross-section of $8 \mathrm{~mm} \times 12 \mathrm{~mm}$ and the thicknesses of the layers being equal to $1 \mathrm{~mm}$, $10 \mathrm{~mm}$ and $2 \mathrm{~mm}$ respectively (see Fig.10). The entirety of the propagating waves can be sought through WFE and without the need of any kinematic assumptions for the complex structures, as 3D FEs and displacement fields are employed. In the general case where a nonlinear stress-strain relation is employed for the mechanics of the coupling element, a dedicated algorithm has to be developed as in [43], in order to compute the nonlinear force vector by inputting the kinematics of each FE. This however is out of the scope of this work, therefore (as with the aluminium beam case study), nonlinear spring elements will be used in combination with linear 3D FEs for which $\mathbf{f}_{N L}$ will be straightforward to compute. 


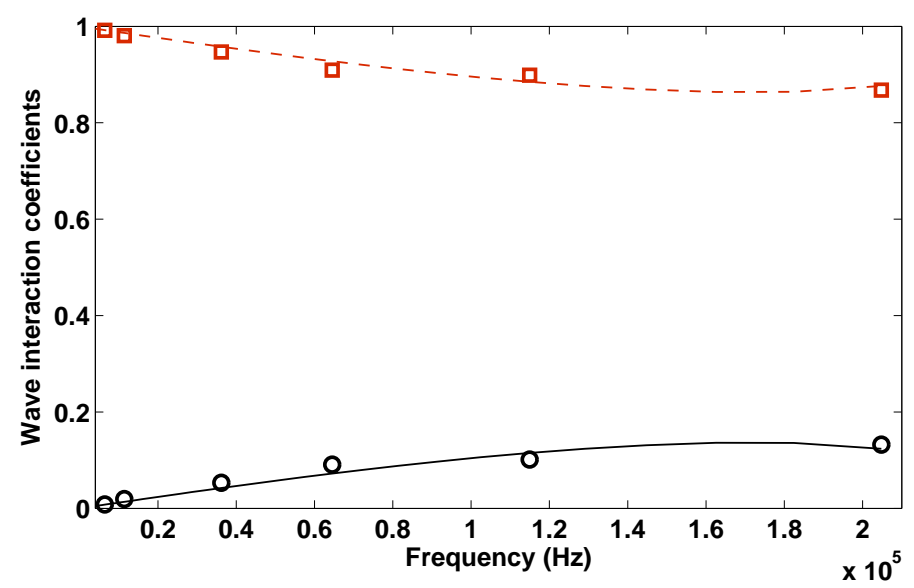

Figure 9: Absolute values of the wave reflection and transmission coefficients for the pressure propagating wave at harmonic frequency $3 \omega$ for a nonlinearly hardened element with $k_{n l}=1 \mathrm{e} 13 \mathrm{~N} / \mathrm{m}^{3}$ : Reflection coefficients computed according to the current scheme (-), Reflection coefficients computed through a full transient FE calculation (o), Transmission coefficients computed according to the current scheme (-,), Transmission coefficients computed through a full transient FE calculation ( $\square$ ).

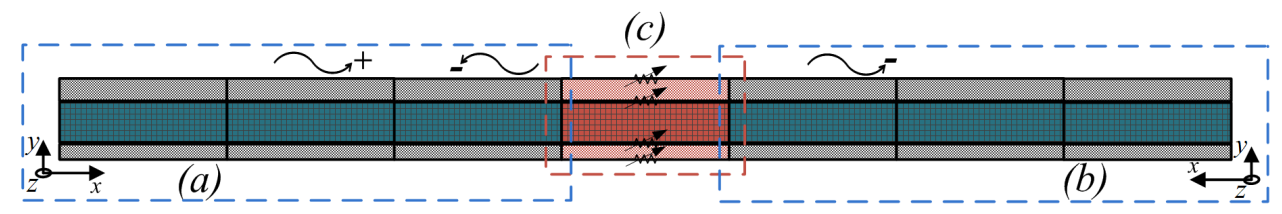

Figure 10: Schematic representation of the two healthy and elastic composite multilayer waveguides (a) and (b) coupled through an element (c) exhibiting structural nonlinearity.

In practice, the wave modes can be excited one-by-one in a full FE transient simulation by employing the WFE computed $\phi_{q, n}^{+}$eigenvectors and applying them as time-dependent harmonic displacement boundary conditions (of excitation frequency $\omega$ ) at one of the extreme cross-sections of the waveguide. An 11-cycle Hanning window was used for all transient excitations. The results on the fundamental and harmonic reflections for a nonlinearly hardening element are presented below in Figs.11. Excellent agreement is observed between the fundamental frequency reflection predictions obtained through the presented approach and through a full FE transient response prediction. Moreover, good agreement is observed between the two approaches regarding the reflections computed for the $3 \omega$ harmonic frequency. The most probable cause of the slight divergence observed between the two sets of results is the fact that in a nonlinear transient FE problem energy is partially also channeled towards other harmonics (other than the second one), which are not included in the harmonic balance expansion.

\section{Conclusions}

A novel comprehensive FE-based computational scheme was presented for quantifying guided wave interaction with LSNs. Layered complex structures can be modelled through the presented approach as an FE discretization is 


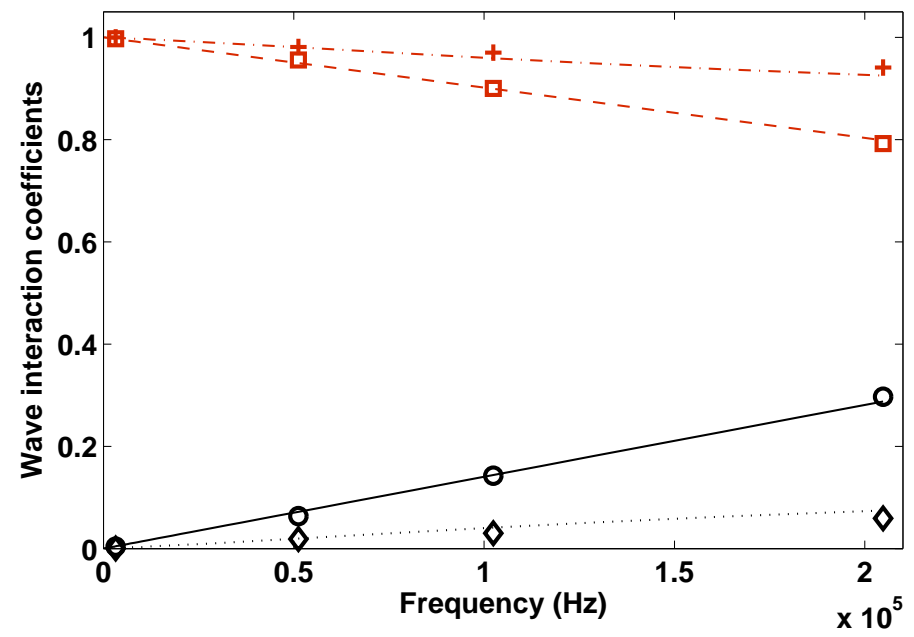

Figure 11: Absolute values of the wave reflection and transmission coefficients of the pressure propagating wave for a nonlinearly hardened element with $k_{n l}=1 \mathrm{e} 13 \mathrm{~N} / \mathrm{m}^{3}$ at: Reflection coefficients at frequency $\omega$ computed according to the current scheme (-), Reflection coefficients at frequency $\omega$ computed through a full transient FE calculation (०), Reflection coefficients at frequency $3 \omega$ computed according to the current scheme ( $\cdots)$, Reflection coefficients at frequency $3 \omega$ computed through a full transient FE calculation $(\diamond)$, Transmission coefficients at frequency $\omega$ computed according to the current scheme (-,), Transmission coefficients at frequency $\omega$ computed through a full transient FE calculation ( $\square$ ), Transmission coefficients at frequency $3 \omega$ computed according to the current scheme (- - ), Transmission coefficients at frequency $3 \omega$ computed through a full transient FE calculation $(+)$.

employed. The scheme couples wave propagation properties within linear structural waveguides to LSNs and is able to compute the generation of harmonic frequencies for each wave mode through a harmonic balance projection. The principal outcomes of the work are summarized as follows:

(i) The presented scheme was validated through comparison with a full $\mathrm{FE}$ transient response prediction. Excellent agreement is observed between the two sets of results for the fundamental, as well as for higher harmonic frequency predictions.

(ii) The new approach is able to predict reflections and transmissions at harmonic frequencies with a speed that is orders of magnitude faster than conventional transient FE solutions. The exhibited approach focuses on calculations for 1D structures with a 2D methodology extension currently under development.

(iii) Generation of higher order harmonics can become maximum at certain frequencies which can be excited for facilitating the detection of certain nonlinearity scenarios (ideally related to the presence of certain damage).

Future developments are focusing towards modelling and implementing realistic damage models as a LSN. Efficient multiscale damage models are essential to develop in order to accurately capture the nonlinear mechanics of advanced damage scenarios, while retaining the size of the FE model and the implied required computational effort at acceptable levels. 


\section{References}

[1] C. Lissenden, Y. Liu, J. Rose, Use of non-linear ultrasonic guided waves for early damage detection, Insight-Non-Destructive Testing and Condition Monitoring 57 (2015) 206-11.

[2] S. S. Kessler, S. M. Spearing, C. Soutis, Damage detection in composite materials using lamb wave methods, Smart Materials and Structures 11 (2002) 269.

[3] M. Breazeale, D. Thompson, Finite-amplitude ultrasonic waves in aluminum, Applied Physics Letters 3 (1963) 77-8.

[4] A. Vakakis, Scattering of structural waves by nonlinear elastic joints, Journal of vibration and acoustics 115 (1993) $403-10$.

[5] A. H. Nayfeh, A. F. Vakakis, T. A. Nayfeh, A method for analyzing the interaction of nondispersive structural waves and nonlinear joints, The Journal of the Acoustical Society of America 93 (1993) 849-56.

[6] P. B. Nagy, Fatigue damage assessment by nonlinear ultrasonic materials characterization, Ultrasonics 36 (1998) $375-81$.

[7] D. M. Donskoy, A. M. Sutin, Vibro-acoustic modulation nondestructive evaluation technique, Journal of intelligent material systems and structures 9 (1998) 765-71.

[8] V. Zaitsev, V. Nazarov, V. Gusev, B. Castagnede, Novel nonlinear-modulation acoustic technique for crack detection, NDT \& E International 39 (2006) 184-94.

[9] V. J. Rao, E. Kannan, R. V. Prakash, K. Balasubramaniam, Fatigue damage characterization using surface acoustic wave nonlinearity in aluminum alloy aa7175-t7351, Journal of Applied Physics 104 (2008) 123508.

[10] H. Hu, W. Staszewski, N. Hu, R. Jenal, G. Qin, Crack detection using nonlinear acoustics and piezoceramic transducersinstantaneous amplitude and frequency analysis, Smart Materials and Structures 19 (2010) 065017.

[11] Z. Su, C. Zhou, M. Hong, L. Cheng, Q. Wang, X. Qing, Acousto-ultrasonics-based fatigue damage characterization: Linear versus nonlinear signal features, Mechanical Systems and Signal Processing 45 (2014) 225-39.

[12] H. J. Lim, H. Sohn, M. P. DeSimio, K. Brown, Reference-free fatigue crack detection using nonlinear ultrasonic modulation under various temperature and loading conditions, Mechanical Systems and Signal Processing 45 (2014) 468-78.

[13] K.-A. Van Den Abeele, P. A. Johnson, A. Sutin, Nonlinear elastic wave spectroscopy (news) techniques to discern material damage, part i: nonlinear wave modulation spectroscopy (nwms), Research in nondestructive evaluation 12 (2000) 17-30.

[14] K. E. Van Den Abeele, A. Sutin, J. Carmeliet, P. A. Johnson, Micro-damage diagnostics using nonlinear elastic wave spectroscopy (news), Ndt \& E International 34 (2001) 239-48.

[15] M. Meo, U. Polimeno, G. Zumpano, Detecting damage in composite material using nonlinear elastic wave spectroscopy methods, Applied composite materials 15 (2008) 115-26.

[16] M. Scalerandi, V. Agostini, P. P. Delsanto, K. Van Den Abeele, P. A. Johnson, Local interaction simulation approach to modelling nonclassical, nonlinear elastic behavior in solids, The Journal of the Acoustical Society of America 113 (2003) 3049-59.

[17] P. P. Delsanto, A. Gliozzi, M. Hirsekorn, M. Nobili, A $2 d$ spring model for the simulation of ultrasonic wave propagation in nonlinear hysteretic media, Ultrasonics 44 (2006) 279-86.

[18] C. Pecorari, Nonlinear interaction of plane ultrasonic waves with an interface between rough surfaces in contact, The Journal of the Acoustical Society of America 113 (2003) 3065-72.

[19] A. J. Croxford, P. D. Wilcox, B. W. Drinkwater, P. B. Nagy, The use of non-collinear mixing for nonlinear ultrasonic detection of plasticity and fatigue, The Journal of the Acoustical Society of America 126 (2009) EL117-22.

[20] V. Giurgiutiu, M. Gresil, B. Lin, A. Cuc, Y. Shen, C. Roman, Predictive modeling of piezoelectric wafer active sensors interaction with high-frequency structural waves and vibration, Acta Mechanica 223 (2012) 1681-91.

[21] Y. Shen, V. Giurgiutiu, Predictive modeling of nonlinear wave propagation for structural health monitoring with piezoelectric wafer active sensors, Journal of Intelligent Material Systems and Structures 25 (2014) 506-20.

[22] T. B. Autrusson, K. G. Sabra, M. J. Leamy, Reflection of compressional and rayleigh waves on the edges of an elastic plate with quadratic nonlinearity, The Journal of the Acoustical Society of America 131 (2012) 1928-37. 
[23] G. M. Fierro, F. Ciampa, D. Ginzburg, E. Onder, M. Meo, Nonlinear ultrasound modelling and validation of fatigue damage, Journal of Sound and Vibration 343 (2015) 121-30.

[24] N. Rauter, R. Lammering, T. Kühnrich, On the detection of fatigue damage in composites by use of second harmonic guided waves, Composite Structures 152 (2016) 247-58.

[25] K.-Y. Jhang, Nonlinear ultrasonic techniques for nondestructive assessment of micro damage in material: a review, International journal of precision engineering and manufacturing 10 (2009) 123-35.

[26] I. Solodov, D. Döring, G. Busse, New opportunities for ndt using non-linear interaction of elastic waves with defects, Strojniški vestnikJournal of Mechanical Engineering 57 (2011) 169-82.

[27] K. Matlack, J.-Y. Kim, L. Jacobs, J. Qu, Review of second harmonic generation measurement techniques for material state determination in metals, Journal of Nondestructive Evaluation 34 (2015) 1-23.

[28] D. Broda, W. Staszewski, A. Martowicz, T. Uhl, V. Silberschmidt, Modelling of nonlinear crack-wave interactions for damage detection based on ultrasounda review, Journal of Sound and Vibration 333 (2014) 1097-118.

[29] D. Mead, A general theory of harmonic wave propagation in linear periodic systems with multiple coupling, Journal of Sound and Vibration 27 (1973) 235-60.

[30] R. Langley, A note on the force boundary conditions for two-dimensional periodic structures with corner freedoms, Journal of Sound and Vibration 167 (1993) 377-81.

[31] B. R. Mace, D. Duhamel, M. J. Brennan, L. Hinke, Finite element prediction of wave motion in structural waveguides, The Journal of the Acoustical Society of America 117 (2005) 2835-43.

[32] D. Duhamel, B. R. Mace, M. J. Brennan, Finite element analysis of the vibrations of waveguides and periodic structures, Journal of Sound and Vibration 294 (2006) 205-20.

[33] J. M. Renno, B. R. Mace, On the forced response of waveguides using the wave and finite element method, Journal of Sound and Vibration 329 (2010) 5474-88.

[34] D. Chronopoulos, M. Ichchou, B. Troclet, O. Bareille, Computing the broadband vibroacoustic response of arbitrarily thick layered panels by a wave finite element approach, Applied Acoustics 77 (2014) 89-98.

[35] D. Chronopoulos, B. Troclet, O. Bareille, M. Ichchou, Modeling the response of composite panels by a dynamic stiffness approach, Composite Structures 96 (2013) 111-20.

[36] D. Chronopoulos, M. Ichchou, B. Troclet, O. Bareille, Predicting the broadband response of a layered cone-cylinder-cone shell, Composite Structures 107 (2014) 149-59.

[37] J.-M. Mencik, M. Ichchou, Multi-mode propagation and diffusion in structures through finite elements, European Journal of MechanicsA/Solids 24 (2005) 877-98.

[38] J. M. Renno, B. R. Mace, Calculation of reflection and transmission coefficients of joints using a hybrid finite element/wave and finite element approach, Journal of Sound and Vibration 332 (2013) 2149-64.

[39] R. Langley, K. Heron, Elastic wave transmission through plate/beam junctions, Journal of sound and vibration 143 (1990) $241-53$.

[40] R. E. Mickens, Truly nonlinear oscillations: harmonic balance, parameter expansions, iteration, and averaging methods, World Scientific, 2010.

[41] R. K. Narisetti, M. Ruzzene, M. J. Leamy, Study of wave propagation in strongly nonlinear periodic lattices using a harmonic balance approach, Wave Motion 49 (2012) 394-410.

[42] F. Moser, L. J. Jacobs, J. Qu, Modeling elastic wave propagation in waveguides with the finite element method, NDT \& E International 32 (1999) 225-34.

[43] K. Manktelow, R. K. Narisetti, M. J. Leamy, M. Ruzzene, Finite-element based perturbation analysis of wave propagation in nonlinear periodic structures, Mechanical Systems and Signal Processing 39 (2013) 32-46. 